\title{
Patient Selection for Epicardial Ablation- Part I: The Role of Epicardial Ablation in Various Cardiac Disease States
}

\author{
JUSTIN A. EDWARD, MD ${ }^{1}$ and DUY T. NGUYEN, MD, FHRS ${ }^{1}$ \\ ${ }^{1}$ Section of Cardiac Electrophysiology, Division of Cardiology, University of Colorado Denver, Aurora, CO, USA
}

\begin{abstract}
Epicardial catheter ablation is most commonly performed following unsuccessful endocardial ablation. Given the frequency of epicardial substrates in certain cardiomyopathic disease states, however, a combined endocardial-epicardial approach should be considered as a primary treatment strategy. Although epicardial ablation is primarily deployed in patients with ventricular arrhythmias, the role of epicardial approaches in supraventricular tachycardias (eg, atrial fibrillation, inappropriate sinus tachycardia, and - rarely-accessory pathways) is growing, with continued advances being made.
\end{abstract}

KEYWORDS. Arrhythmia, catheter, epicardial, radiofrequency ablation.
ISSN 2156-3977 (print) ISSN 2156-3993 (online) CC BY 4.0 license

(C) 2019 Innovations in Cardiac Rhythm Management

\section{Introduction}

Epicardial catheter ablation is most commonly relied on for the management of arrhythmogenic substrates arising outside of the endocardium. Most patients considered for epicardial ablation have already undergone an attempted endocardial catheter ablation. The sites of origin for various arrhythmias, particularly ventricular tachycardia (VT), are not limited to the subendocardial myocardium. In particular, patients with nonischemic cardiomyopathy can have either intramural or subepicardial substrates that give rise to VT. Epicardial catheter ablation for the treatment of VT is often required when critical elements of VT circuits are epicardial in origin. ${ }^{1}$

Dr. Nguyen receives significant research grants from Biosense Webster and CardioNXT and educational grants from Biosense Webster, Abbott, Boston Scientific, and Medtronic. Dr. Nguyen has provisional patents on partially insulated focused catheter ablation and facilitated ablation. Dr. Nguyen has nonpublic equity interests / stock options in CardioNXT. Dr. Edward reports no conflicts of interest for the published content.

Manuscript received March 4, 2019. Final version accepted March 29, 2019.

Address correspondence to: Duy T. Nguyen, MD, Section of Cardiac Electrophysiology, University of Colorado, B-132, Leprino Building, 12401 East $17^{\text {th }}$ Avenue, Aurora, CO 80045, USA.

Email: duy.t.nguyen@ucdenver.edu.
The technique of percutaneous epicardial mapping and ablation was first described in patients with Chagas cardiomyopathy as a means to ablate VT more than 20 years ago. ${ }^{1}$ Prior to the development of epicardial catheter ablation, patients with ventricular arrhythmias that were nonresponsive to endocardial ablation often required surgical intervention.

Similar advances have been made for patients experiencing symptomatic supraventricular tachycardias (SVTs), including drug-refractory atrial fibrillation (AF). Pulmonary vein (PV) isolation is recognized as the cornerstone of AF ablation. ${ }^{2}$ Previously, catheter ablation of AF has been limited to the right atrial (RA) and left atrial (LA) endocardia, and only surgical approaches have employed epicardial AF ablation access. ${ }^{3,4}$ In patients with recurrent AF despite PV isolation, additional arrhythmia sources outside of the PV antrum are suspected. More recently, hybrid procedures combining epicardial and endocardial ablation for AF have been utilized and have demonstrated better efficacy than isolated ablation procedures. ${ }^{5}$ This paper aims to review the indications, techniques, and developments in the field of epicardial catheter ablation as well as to highlight the unique subset of patients in whom epicardial ablation may be indicated. 


\section{Indications for epicardial catheter ablation in ventricular tachycardia}

Epicardial ablation is most commonly performed following unsuccessful endocardial ablation of midmyocardial or epicardial substrates. However, it may also be considered based on the etiology of the underlying disease process, substrate localization on imaging, specific electrocardiographic (ECG) criteria, or prior mapping demonstrating circuits of epicardial origin. In these cases, a combined endocardial-epicardial approach is associated with improved freedom from VT recurrence when compared with limited endocardial strategies.

\section{Epicardial ventricular tachycardia in various cardiomyopathic disease states}

Although the use of epicardial ablation has previously been less common, recently, at high-volume centers, it is becoming a more routine procedure. Of the information available prior to VT ablation, the type of cardiomyopathy is probably the best predictor for determining the necessity of an epicardial approach. Epicardial ablation should be considered when there is a high pretest probability of midmyocardial or subepicardial scar, which is commonly seen in patients with idiopathic dilated cardiomyopathy (IDCM), arrhythmogenic right ventricular (RV) cardiomyopathy (ARVC), and hypertrophic cardiomyopathy (HCM). In particular types of cardiomyopathy, however, it is not only important to determine if epicardial ablation needs to be performed but to also identify when a combination of endocardial and epicardial ablation is warranted (Table 1).

\section{Idiopathic dilated cardiomyopathy}

The prevalence of epicardial VT in patients with nonischemic dilated cardiomyopathy is higher in comparison with in those with an ischemic VT substrate. ${ }^{6,7}$ In patients with IDCM, the main mechanism of VT is myocardial reentry. Pathological arrhythmogenic foci often originate at sites of scar tissue over the basal lateral left ventricle (LV) adjacent to mitral and aortic valve annuli deep in the endocardium. ${ }^{6}$ Areas of scar, however, may be larger in the epicardium than in the endocardium and, in some patients, are entirely limited to the epicardial surface, giving rise to arrhythmias that cannot be ablated via an endocardial approach. Contrast-enhanced cardiac magnetic resonance imaging (MRI) in patients with IDCM reveals midwall myocardial fibrosis in about one-third of cases, a finding that has been suggested as predictive of future arrhythmic events. ${ }^{8}$ Intracardiac echocardiography (ICE) has also been used to identify increased echogenicity in the lateral wall of the LV, which has been found to correspond with abnormal epicardial substrate and thereby guide an epicardial approach. ${ }^{9}$ Combined endocardial and epicardial catheter ablation in patients with IDCM and VT has achieved high rates of arrhythmia-free outcomes. ${ }^{10,11}$ Many patients in these studies had previously failed prior endocardial ablation. Thus,

Table 1: Prevalence of Epicardial VT Origins in Various Cardiomyopathic Disease States

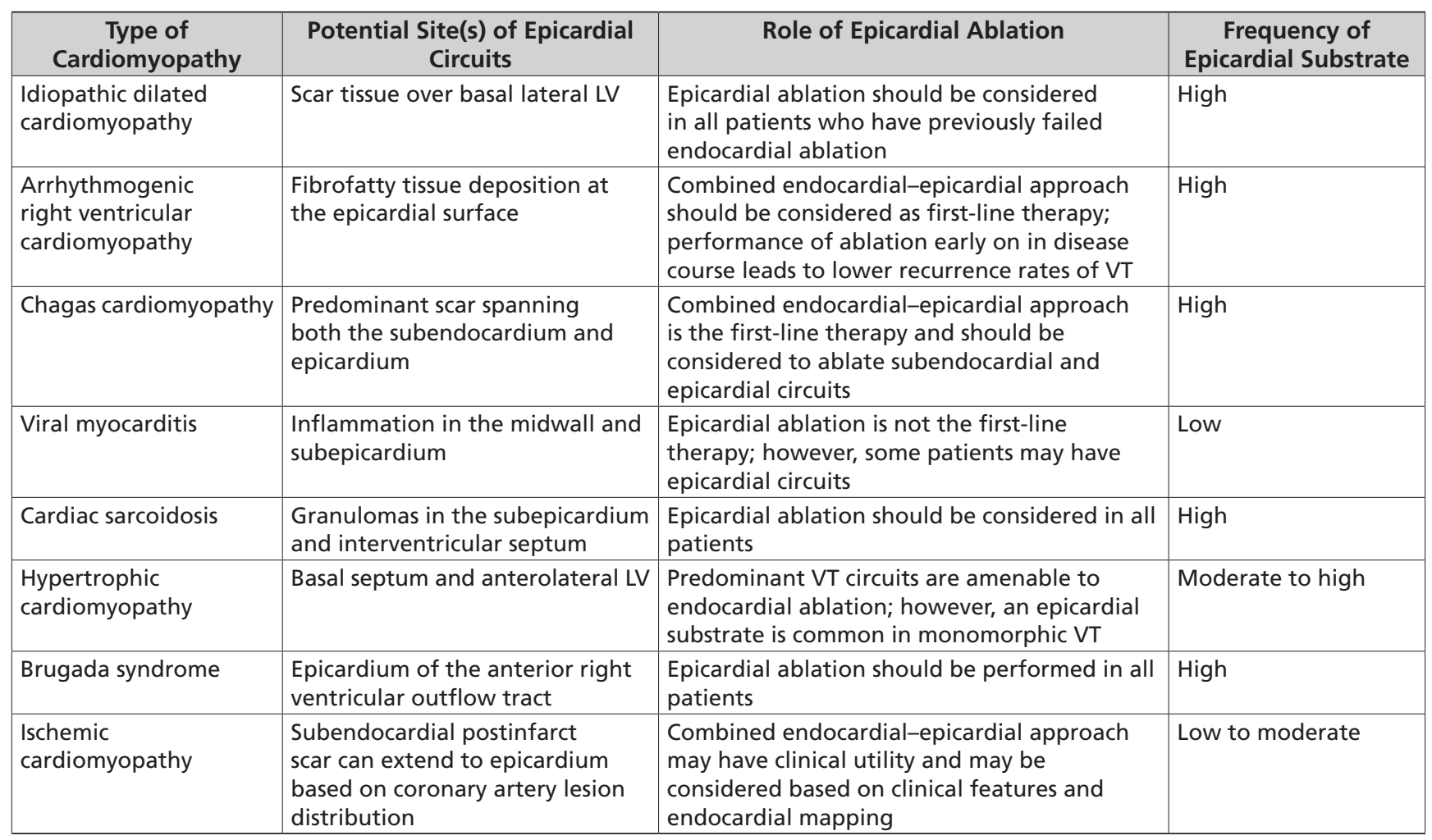

LV: left ventricle; VT: ventricular tachycardia. 
although choosing epicardial ablation prior to an endocardial approach in patients with IDCM may not always be the first-line option, epicardial ablation should be considered for all patients who previously failed endocardial ablation.

\section{Arrhythmogenic right ventricular cardiomyopathy}

ARVC is an inherited disease characterized by fibrofatty tissue replacement of the myocytes predominantly in the RV. Early studies with endocardial ablation alone of VT in ARVC patients yielded suboptimal results, with almost half of patients experiencing arrhythmia recurrence despite taking antiarrhythmic drugs. ${ }^{12}$ An autopsy study of patients with ARVC found increased fibrofatty tissue deposition at the epicardial surface and, thus, epicardial circuits are generally indicated as the source of arrhythmia in these individuals. ${ }^{13}$ Accordingly, concomitant endocardial and epicardial mapping in ARVC patients revealed greater areas of low-voltage electrograms on the epicardial surface. ${ }^{14}$ The use of epicardial ablation as an adjunctive strategy with an endocardial approach has been reported in many observational studies of ARVC, and combined endocardial-epicardial mapping and ablation has been shown to reduce VT recurrence. ${ }^{14-16} \mathrm{~A}$ meta-analysis comparing combined endocardial-epicardial VT ablation and an endocardial-alone approach in ARVC patients found higher rates of freedom from recurrent ventricular arrhythmias or implantable cardioverter-defibrillator (ICD) placement with the former. ${ }^{17}$ Often, to prevent VT recurrence in ARVC patients, both endocardial and epicardial ablation should be performed.

\section{Chagas cardiomyopathy}

The first described study of percutaneously accessing the pericardial space for epicardial VT ablation involved patients with Chagas cardiomyopathy. ${ }^{1}$ It was later reported that deploying combined endocardial-epicardial VT ablation in these patients could achieve higher arrhythmia-free success rates. ${ }^{18}$ Epicardial ablation is most likely beneficial in this specific group of patients because it has been shown that patients with Chagas cardiomyopathy and VT have an approximately twofold greater epicardial versus endocardial scar area. ${ }^{19}$ Due to the presence of subepicardial circuits, a combined endocardial-epicardial approach should be used in patients with Chagas cardiomyopathy in order to ablate reentrant circuits spanning both the subendocardium and epicardium.

\section{Viral myocarditis}

Outside of the realm of Chagas myocarditis, there have been very few reported cases demonstrating the role of epicardial VT ablation for myocarditis. The application of contrast-enhanced cardiac MRI in patients with active myocarditis, however, has revealed inflammation present in the midwall and subepicardium, lending support to the notion that epicardial ablation may have an important role in ablating VT in myocarditis patients. ${ }^{20,21}$ In a study of 20 patients with drug-refractory VT and biopsy-proven viral myocarditis, approximately one-third of patients required epicardial ablation to successfully eliminate the arrhythmia. ${ }^{22}$ As viral myocarditis is often a self-limiting process, the current data do not support the role of epicardial ablation in all patients with myocarditis, as VT can often cease spontaneously during recovery.

\section{Cardiac sarcoidosis}

Patients with cardiac sarcoidosis also boast a significant epicardial substrate with a predilection of granulomas in the subepicardium and interventricular septum. ${ }^{23}$ In a systematic review of five VT ablation studies in cardiac sarcoidosis patients, approximately $20 \%$ of the total 83 patients required epicardial ablation. ${ }^{24}$ VT burden is high in cardiac sarcoidosis patients, with a mean of three VTs per patient. It is therefore not surprising that the rate of freedom from VT recurrence was only $50 \%$ at a median follow-up point of 24 months, with about one-fourth of patients having to undergo redo procedures. ${ }^{24,25}$

\section{Hypertrophic cardiomyopathy}

In HCM, monomorphic VT with an epicardial substrate may be present in up to $80 \%$ of affected patients. ${ }^{26}$ Although the predominant VT circuits are distributed in the basal septum and anterolateral LV and may be amenable to endocardial ablation, epicardial VT mapping and ablation should be considered in patients with refractory VT. ${ }^{27,28}$ Epicardial ablation in HCM is mostly pertinent for patients with apical aneurysms, which compose a minority of individuals with HCM. Most patients with septal hypertrophy have arrhythmias that are not approachable from the epicardium.

\section{Brugada syndrome}

In contrast with other etiologies of cardiomyopathy, Brugada syndrome gives rise to ventricular arrhythmias in the epicardium of the anterior RV outflow tract, and epicardial ablation of this pathological substrate can render VT noninducible and provide long-term arrhythmia-free success. ${ }^{29}$ In patients with Brugada syndrome who underwent both endocardial and epicardial mapping, there was no identifiable endocardial substrate in $93 \%$ of cases, thus supporting the notion that an epicardial approach would favor freedom from recurrent ventricular arrhythmias. ${ }^{30}$

\section{Ischemic cardiomyopathy}

In patients with ischemic cardiomyopathy, postinfarct scar is located in the subendocardium and can extend to the epicardium depending upon the patient's specific coronary artery distribution. The arrhythmogenic tissue can be accessed via the endocardium, and it has been commonly thought that epicardial ablation strategies have lower yield in postinfarction patients. ${ }^{31}$ The 
prevalence of epicardial VT in patients with a history of myocardial infarction ranges from $14 \%$ to $33 \% .{ }^{32}$ A recent study of patients with ischemic cardiomyopathy found abnormal epicardial substrates in $14 \%$ of patients. ${ }^{33}$ In a large series of patients with postinfarction VT, epicardial ablation was primarily performed after failed endocardial ablation and an epicardial approach was successful in abolishing the VT circuit in approximately $6 \%$ of the population. ${ }^{34}$ A combined endocardial-epicardial ablation strategy resulted in higher rates of VT-free survival after almost three years of follow-up as compared with endocardial ablation alone. ${ }^{33}$ In patients with ischemic cardiomyopathy, one must consider not only ablation history but also evidence of abnormal epicardial substrate with fractionated or split electrograms that may be visualized during epicardial mapping; these patients are those who may be ideal candidates for combined endocardial-epicardial ablation.

\section{Imaging modalities for epicardial arrhythmogenic substrates}

An unsuccessful endocardial ablation can suggest that the offending substrate may not be accessible via an endocardial approach. In this subset of patients, preprocedural imaging can be particularly useful if it demonstrates or helps to identify epicardial substrates. As such, understanding the underlying arrhythmogenic substrate leading to VT is essential in achieving a successful ablation procedure.

Contrast-enhanced cardiac MRI is considered the gold standard to delineate three-dimensional scar tissue. The location of the scar on preprocedural MRI can help guide one to employ an endocardial and /or epicardial approach in order to reach the VT circuit. The location of scar may help to predict the location of a critical VT isthmus. ${ }^{35}$ In patients with nonischemic cardiomyopathy, scar tissue is most commonly located either intramurally or on the epicardial surface. However, the lowest rates of successful ablation have been reported in patients in whom the majority of scar had an intramural distribution. ${ }^{35}$ In patients with ARVC, the electrical substrate most often originates in the epicardium, likely because the disease process begins at this myocardial surface. MRI can be integrated with electroanatomical mapping to provide higher resolution for the detection and localization of nontransmural scar as compared with other imaging modalities. ${ }^{36}$ It must be noted, however, that the most important limitation of MRI is its use in cardiac device recipients, as the majority of patients undergoing VT ablation also have an ICD. Although ICDs may not necessarily preclude MRI given recent studies and improved MRI compatbility, ${ }^{37,38}$ imaging of the heart may be impaired due to artifacts created by the pulse generator or lead/ coil. ${ }^{39}$

ICE provides real-time, two-dimensional anatomical information and allows for the monitoring of catheter-tissue contact, lesion formation, and potential complications without the risk of radiation exposure from other imaging methods. ${ }^{40}$ In addition, it may have a role in identifying epicardial scar as VT substrate. In a study of patients with nonischemic cardiomyopathy with recurrent VT and abnormal echogenicity on ICE imaging, it was demonstrated that areas of increased echogenicity in the lateral wall corresponded with the abnormal epicardial substrate seen on electroanatomic mapping when reviewed by a blinded echocardiographer. ${ }^{9}$ These abnormal areas of increased echogenicity were absent in the control group, who had structurally normal hearts.

\section{Role of electrocardiogram criteria in epicardial ventricular tachycardia}

ECG criteria may be clinically useful when assessing for the presence of epicardial substrate. Several groups have used 12-lead ECG tracings to investigate specific characteristics that may be helpful prior to the electrophysiological study in determining the epicardial origin of VT. One of the first studies, published by Berruezo et al., suggested the following three ECG criteria are able to identify the epicardial origin of VT with good sensitivity and specificity: a pseudodelta wave of $34 \mathrm{~ms}$ or more (sensitivity: $83 \%$; specificity: $95 \%$ ), an intrinsicoid deflection time of 85 ms or more (sensitivity: $87 \%$; specificity: $90 \%$ ), and an R/S complex duration of $121 \mathrm{~ms}$ or more (sensitivity: $76 \%$; specificity: $85 \%){ }^{41}$

A subsequent study by Bazan et al. suggested different site-specific ECG criteria for LV VT based on the initial vector of the QRS complex. ${ }^{42}$ Specifically, Bazan et al. found that the ECG criteria proposed by Berruezo et al. had varying degrees of specificity and sensitivity depending not only on the type of structural heart disease but also on different sites within the LV, which can vary in terms of local circuit activation. Site-specific morphology criteria support that the following must be present for epicardial LV VT: a Q-wave in lead I for basal superior and apical superior sites, the absence of a Q-wave in any of the inferior leads for basal superior sites, and a Q-wave in inferior leads for basal inferior and apical inferior sites. These proposed morphology criteria correctly identified epicardial origin in $84 \%$ of VT cases. $^{42}$ Based on prior studies, Vallès et al. proposed an algorithm to identify epicardial VT originating from the basal superior and lateral LV in patients with nonischemic cardiomyopathy. ${ }^{43}$ The absence of a Q-wave in the inferior leads with either a pseudo-delta wave of more than $75 \mathrm{~ms}$, a maximum deflection index of more than 0.59 , and/or the presence of a Q-wave in lead I are indicative of epicardial origin with both a sensitivity and specificity of more than $90 \% .{ }^{43}$

These criteria have proven to be optimal parameters to guide the recognition of an epicardial source primarily in patients with nonischemic cardiomyopathy.

ECG criteria can be helpful in determining potential circuits of epicardial VT origin; however, one must take into account the type of cardiomyopathy and the imaging of potential VT substrate when applying the previously mentioned ECG criteria in the assessment of VT. 
Table 2: Clinical Features Suggestive of Epicardial VT Origins

\begin{tabular}{|c|c|c|c|}
\hline Underlying Substrate & ECG Findings & Imaging & Endocardial Mapping \\
\hline \multirow[t]{2}{*}{$\begin{array}{l}\text { Cardiomyopathies } \\
\text { (as listed in Table 1) }\end{array}$} & $\begin{array}{l}\text { NICM: } \\
\text { - Absence of inferior Q-waves } \\
\text { - Pseudo-delta wave > } 75 \mathrm{~ms} \\
\text { - MDI > } 0.59 \\
\text { - Presence of Q-wave in lead I }\end{array}$ & \multirow{2}{*}{$\begin{array}{l}\text { Subepicardial or } \\
\text { midmyocardial } \\
\text { scar detected } \\
\text { either on } \\
\text { contrast-enhanced } \\
\text { computed } \\
\text { tomography } \\
\text { or magnetic } \\
\text { resonance } \\
\text { imaging }\end{array}$} & \multirow{2}{*}{$\begin{array}{l}\text { - Diffuse early activation } \\
\left.\text { (> } 2 \mathrm{~cm}^{2} \text { sites in } 10 \mathrm{~ms}\right) \\
\text { - Far-field early EGM } \\
\text { followed by near-field EGM } \\
\text { - Inability to capture far-field } \\
\text { component of earliest EGM } \\
\text { - Unipolar voltage suggestive } \\
\text { of epicardial scar }\end{array}$} \\
\hline & $\begin{array}{l}\text { ICM: } \\
\text { - Pseudo-delta wave > } 34 \mathrm{~ms} \\
\text { - IDT > } 85 \mathrm{~ms} \\
\text { - Shortest RS complex > } 121 \mathrm{~ms} \\
\text { - QRS duration }>211 \mathrm{~ms}\end{array}$ & & \\
\hline
\end{tabular}

ECG: electrocardiogram; EGM: electrogram; IDT: intrinsicoid deflection time; MDI: maximum deflection index.

\section{Endocardial mapping}

ECG criteria are not always reliable in diagnosing epicardial VT and, in some cases, detailed endocardial mapping can be predictive of epicardial VT circuits (Table 2). In one particular study of patients with VT who were referred for ablation, bipolar electrograms (EGMs) in areas of early endocardial activation were compared with areas of early epicardial activation using electroanatomic activation mapping. ${ }^{44}$ Three characteristics were found from endocardial mapping that consistently indicated the need for epicardial ablation: (1) diffuse early activation ( $>2 \mathrm{~cm}^{2}$ region of sites equally having their earliest activation within $10 \mathrm{~ms}$ ); (2) the sequence of a far-field EGM followed by a near-field EGM in the region of earliest endocardial activation; and (3) the inability to capture the far-field component of the earliest EGM (stim-QRS interval < EGM-QRS time) or reproduce morphological features of the ventricular arrhythmia complex with stimulation at the earliest endocardial site of activation.

In patients with nonischemic cardiomyopathy and VT, endocardial unipolar low-voltage areas were effective in identifying regions with epicardial bipolar low voltages in $60 \%$ of cases.$^{45}$ One study confirmed that low endocardial unipolar voltages of below $4.0 \mathrm{mV}$ are independent predictors of epicardial bipolar scar with $80 \%$ accuracy (and $71 \%$ sensitivity and $75 \%$ specificity) ${ }^{46}$ Endocardial mapping has also been found to be predictive of epicardial VT substrates in patients with both ARVC and ischemic cardiomyopathy. ${ }^{33,47}$

\section{Epicardial catheter ablation in supraventricular tachycardias}

An epicardial approach can also be used for SVT ablation; however, it is most commonly employed following unsuccessful endocardial ablation.

\section{Accessory pathways}

Epicardial pathway circuits have been implicated in up to $8 \%$ of failed endocardial ablations of accessory pathways. ${ }^{48}$ In a series of 10 patients with accessory pathways and previously failed endocardial ablations, the earliest activation was recorded epicardially in five cases with a variety of accessory pathway locations including the right atrial appendage to right ventricle, left posteroseptal region, and right posterolateral region. ${ }^{49}$ At two years of follow-up, one patient showed a recurrence of arrhythmia and was successfully reablated. Additional small cases series have been reported of successful epicardial ablation of accessory pathways following failed initial endocardial ablation. ${ }^{50,51}$ In a separate study of 21 patients referred for accessory pathway epicardial ablation after a median of two prior failed endocardial procedures, almost $30 \%$ of the patients had evidence of early epicardial activation. ${ }^{52}$ Extensive epicardial mapping followed by a combined endocardial-epicardial ablation procedure was effective in eliminating the accessory pathway. In patients who failed to show a benefit after endocardial ablation for accessory pathways, epicardial mapping followed by simultaneous endocardal-epicardial ablation should be considered. ${ }^{53}$

\section{Atrial fibrillation}

As is true with epicardial ablation for VT, epicardial catheter ablation in AF is often attempted after failed endocardial PV isolation. Surgical approaches such as the Cox maze procedure were first introduced more than 25 years ago to treat patients with refractory $\mathrm{AF}^{54}$ Although successful in reducing the recurrence of $\mathrm{AF}$, this surgical procedure requires completion of a sternotomy. Over the last 15 years, there has been a push toward minimally invasive procedures and the introduction of thorascopic approaches has led to the replacement of prior cut-andsew lesions. ${ }^{55}$ Surgical ablation is often used to treat AF in patients in whom medications and catheter ablation have failed. Currently, the two primary types of surgical ablation are convergent (or hybrid) ablation and Cox maze IV ablation. The Cox maze IV procedure, first developed in 2002, is a modification from prior procedures in that it incorporates bipolar radiofrequency ablation and cryoablation. However, it still requires sternotomy and the continued need for cardiopulmonary bypass. This procedure had one-year success rates approaching $90 \%$, with more than half of patients presenting with either persistent or long-standing $\mathrm{AF}^{56}$ Five-year outcomes following the Cox maze IV procedure suggest rates of $78 \%$ and $66 \%$ for 
overall freedom from $\mathrm{AF}$ and freedom from $\mathrm{AF}$ while off antiarrhythmic drugs, respectively. ${ }^{57} \mathrm{~A}$ single-center, propensity-matched study reported 10-year survival rates in patients following Cox maze IV and those with untreated AF of $62 \%$ and $42 \%$, respectively. ${ }^{58}$

In a collaboration between cardiothoracic surgeons and electrophysiologists, percutaneous endocardial catheter mapping and ablation were first added to epicardial thorascopic ablation with the hope of increasing the success rates by combining potential advantages of each separate procedure. ${ }^{59}$ This hybrid AF procedure allowed for fast epicardial placement of extensive linear lesions with the endovascular validation of complete bidirectional conduction block. The convergent ablation procedure is different from previous hybrid ablations in that it employs a novel device to perform epicardial ablation via a transdiaphragmatic approach. Single-center studies over the past 10 years have noted success rates of greater than $80 \%$ in restoring sinus rhythm in patients with persistent AF. ${ }^{60-62}$ Furthermore, among patients followed for up to four years after the procedure, sinus rhythm was maintained in more than $80 \%$ of cases. ${ }^{63,64}$ However, although efficacious in restoring sinus rhythm, a recent meta-analysis of prior studies raises concerns about the use of the convergent procedure due to a complication rate of $9 \%$ and a procedural mortality rate of $1.7 \%{ }^{65}$

Isolated epicardial ablation for recurrent atrial arrhythmias has been shown to give rise to iatrogenic circuits with predisposition to atrial flutter., ${ }^{5,66}$ This potential risk of arrhythmias has been thought to be due to the limitations of current ablation techniques in creating transmural lesion sets when applied endoscopically on the epicardium of the beating heart. ${ }^{67}$ The ideal ablation strategy for recurrent $\mathrm{AF}$ would be to create durable transmural lesion sets in a minimally invasive fashion as well as to map out other potential arrhythmogenic foci, thus reducing the possible risk of arrhythmia recurrence. ${ }^{68}$ More recently, however, a combined endocardial and subxiphoid epicardial catheter approach has been used to both map and ablate recurrent AF. ${ }^{69}$ Potential advantages of this new approach include that it is less invasive than thorascopic epicardial ablation and it also provides transmural ablation lines while mapping potential additional arrhythmia mechanisms. These recent studies suggest that, perhaps, with appropriate patient selection and the accurate identification of patient-specific mechanisms, this novel approach may provide an advantage over contemporary techniques with regard to improving patient outcomes after $\mathrm{AF}$ ablation and preventing the recurrence of atrial arrhythmias.

\section{Inappropriate sinus tachycardia}

Successful epicardial ablation has also been reported for inappropriate sinus tachycardia (IST). Sinus node modification with endocardial catheter ablation for the treatment of IST remains a challenge due to the location of the phrenic nerve. While this concern may be greater when employing an epicardial approach given the proximity of the phrenic nerve to the epicardial surface of the heart, the epicardial approach allows for mechanical displacement of the phrenic nerve and therefore safer delivery of energy to the sinus node either epicardially or endocardially (Figure 1). ${ }^{70}$

There are some adverse effects that can present with sinus node ablation, including right diaphragmatic paralysis, transient superior vena cava syndrome, and complete sinus node dysfunction requiring pacing. ${ }^{71}$ More recently, in a case series of five patients who had previously failed endocardial ablation, combined endocardial-epicardial sinus node ablation was found to be successful in resolving symptoms, although three patients did develop postoperative pericarditis. ${ }^{72}$ Several nonrandomized studies have assessed the efficacy of radiofrequency catheter ablation
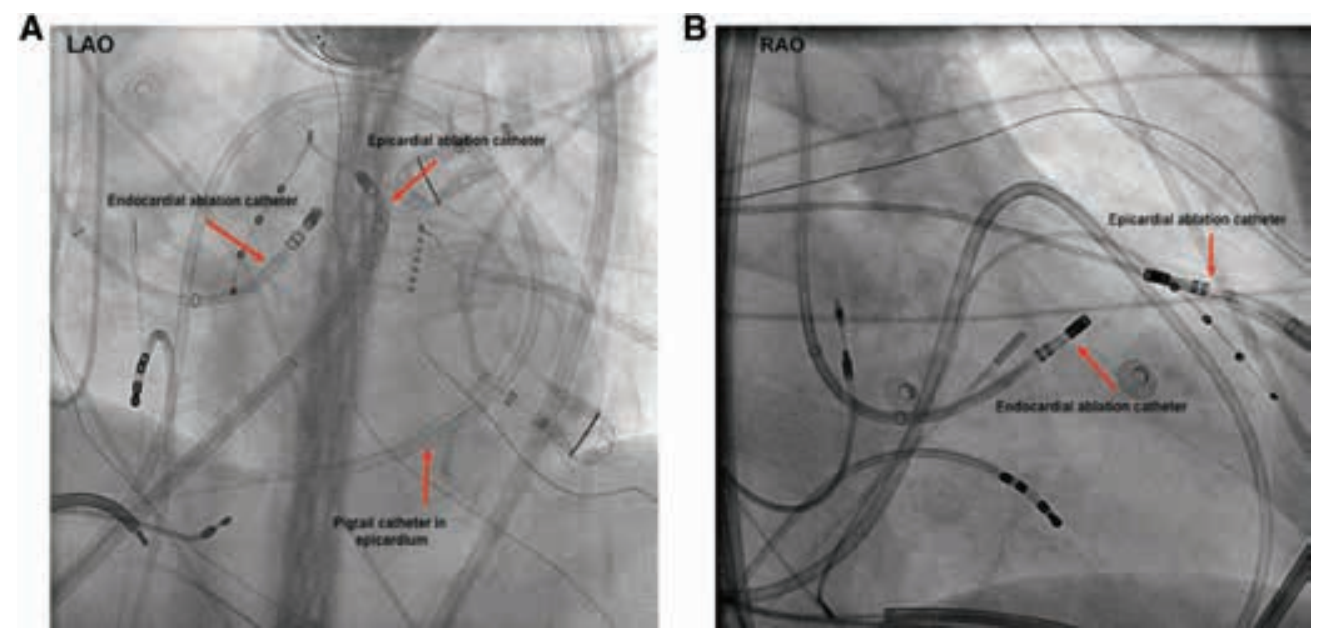

Figure 1: Endocardial-epicardial bipolar ablation configuration. Bipolar ablation across an LV summit midmyocardial circuit may require epicardial access for one of the ablation poles. A and B: Fluoroscopic views (anteroposterior) of two ablation catheters in a bipolar configuration, targeting the LV summit area for this patient. LAO: left anterior oblique; RAO: right anterior oblique. 
with varying degrees of success. ${ }^{49,73-75}$ A meta-analysis of nine prior ablation studies highlighted acute procedural success rates approaching 90\%; however, only one study utilizing an epicardial approach was included. ${ }^{76} \mathrm{In}$ addition, $8.5 \%$ of patients in this study had severe procedural complications (eg, diaphragmatic paralysis, arteriovenous fistula, retroperitoneal bleed) and almost $10 \%$ of patients required pacemaker implantation. ${ }^{76}$ Additional studies need to be performed to determine the safety and efficacy of endocardial-epicardial ablation in patients with refractory IST $^{77}$ In many cases of IST, the risks of ablation may outweigh the benefits, and the procedure is not currently recommended in the guidelines as first-line therapy.

\section{Conclusion}

Many factors play a role in the selection of patients for epicardial mapping and ablation as well as in the safe and effective delivery of ablation lesions in the epicardium; above, we have touched upon a few of the criteria and ablation parameters in this review. Understanding the VT substrate is important in those with nonischemic cardiomyopathy because the epicardium is commonly the site of arrhythmogenic foci. Furthermore, the role of an epicardial approach in patients with SVT is ever-expanding with the novel technological advances ongoing in catheter ablation.

\section{References}

1. Sosa E, Scanavacca M, d'Avila A, Pilleggi F. A new technique to perform epicardial mapping in the electrophysiology laboratory. J Cardiovasc Electrophysiol. 1996;7(6):531-536.

2. Calkins H, Hindricks G, Cappato R, et al. 2017 HRS/EHRA/ ECAS/APHRS/SOLAECE expert consensus statement on catheter and surgical ablation of atrial fibrillation. Europace. 2018;20(1):e1-e160.

3. Pison L, La Meir M, van Opstal J, Blaauw Y, Maessen J, Crijns HJ. Hybrid thoracoscopic surgical and transvenous catheter ablation of atrial fibrillation. J Am Coll Cardiol. 2012;60:(1)54-61.

4. Gehi AK, Mounsey JP, Pursell I, et al. Hybrid epicardial-endocardial ablation using a pericardioscopic technique for the treatment of atrial fibrillation. Heart Rhythm. 2013;10(1):22-28.

5. Gelsomino S, Van Breugel HN, Pison L, et al. Hybrid thoracoscopic and transvenous catheter ablation of atrial fibrillation. Eur J Cardiothorac Surg. 2014;45(3):401-407.

6. Soejima K, Stevenson WG, Sapp JL, Selwyn AP, Couper G, Epstein LM. Endocardial and epicardial radiofrequency ablation of ventricular tachycardia associated with dilated cardiomyopathy: the importance of low-voltage scars. J Am Coll Cardiol. 2004;43(10):1834-1842.

7. Della Bella P, Brugada J, Zeppenfeld K, et al. Epicardial ablation for ventricular tachycardia: a European multicenter study. Circ Arrhythm Electrophysiol. 2011;4(5):653-659.

8. Assomull RG, Prasad SK, Lyne J, et al. Cardiovascular magnetic resonance, fibrosis, and prognosis in dilated cardiomyopathy. J Am Coll Cardiol. 2006;48(10):1977-1985.

9. Bala R, Ren JF, Hutchinson MD, et al. Assessing epicardial substrate using intracardiac echocardiography during VT ablation. Circ Arrhythm Electrophysiol. 2011;4(5):667-673.
10. Cano O, Hutchinson M, Lin D, et al. Electroanatomic substrate and ablation outcome for suspected epicardial ventricular tachycardia in left ventricular nonischemic cardiomyopathy. J Am Coll Cardiol. 2009;54(9):799-808.

11. Nakahara S, Tung R, Ramirez RJ, et al. Characterization of the arrhythmogenic substrate in ischemic and nonischemic cardiomyopathy implications for catheter ablation of hemodynamically unstable ventricular tachycardia. J Am Coll Cardiol. 2010;55(21):2355-2365.

12. Verma A, Kilicaslan F, Schweikert RA, et al. Short- and longterm success of substrate-based mapping and ablation of ventricular tachycardia in arrhythmogenic right ventricular dysplasia. Circulation. 2005;111(24):3209-3216.

13. Basso C, Thiene G, Corrado D, Angelini A, Nava A, Valente M. Arrhythmogenic right ventricular cardiomyopathy. Dysplasia, dystrophy, or myocarditis? Circulation. 1996;94(5):983-991.

14. Garcia FC, Bazan V, Zado ES, Ren JF, Marchlinski FE. Epicardial substrate and outcome with epicardial ablation of ventricular tachycardia in arrhythmogenic right ventricular cardiomyopathy/dysplasia. Circulation. 2009;120(5):366-375.

15. Berruezo A, Fernandez-Armenta J, Mont L, et al. Combined endocardial and epicardial catheter ablation in arrhythmogenic right ventricular dysplasia incorporating scar dechanneling technique. Circ Arrhythm Electrophysiol. 2012;5(1):111-121.

16. Pokushalov E, Romanov A, Turov A, Artyomenko S, Shirokova N, Karaskov A. Percutaneous epicardial ablation of ventricular tachycardia after failure of endocardial approach in the pediatric population with arrhythmogenic right ventricular dysplasia. Heart Rhythm. 2010;7(10):1406-1410.

17. Bai R, Di Biase L, Shivkumar K, et al. Ablation of ventricular arrhythmias in arrhythmogenic right ventricular dysplasia/ cardiomyopathy: arrhythmia-free survival after endo-epicardial substrate based mapping and ablation. Circ Arrhythm Electrophysiol. 2011;4(4):478-485.

18. Sosa E, Scanavacca M, D'Avila A, et al. Endocardial and epicardial ablation guided by nonsurgical transthoracic epicardial mapping to treat recurrent ventricular tachycardia. $J$ Cardiovasc Electrophysiol. 1998;9(3):229-239.

19. Henz BD, do Nascimento TA, Dietrich Cde O, et al. Simultaneous epicardial and endocardial substrate mapping and radiofrequency catheter ablation as first-line treatment for ventricular tachycardia and frequent ICD shocks in chronic chagasic cardiomyopathy. I Interv Card Electrophysiol. 2009;26(3):195-205.

20. De Cobelli F, Pieroni M, Esposito A, et al. Delayed gadolinium-enhanced cardiac magnetic resonance in patients with chronic myocarditis presenting with heart failure or recurrent arrhythmias. J Am Coll Cardiol. 2006;47(8):1649-1654.

21. Goitein O, Matetzky S, Beinart R, et al. Acute myocarditis: noninvasive evaluation with cardiac MRI and transthoracic echocardiography. AJR Am J Roentgenol. 2009;192(1):254-258.

22. Dello Russo A, Casella M, Pieroni M, et al. Drug-refractory ventricular tachycardias after myocarditis: endocardial and epicardial radiofrequency catheter ablation. Circ Arrhythm Electrophysiol. 2012;5(3):492-498.

23. Tavora F, Cresswell N, Li L, Ripple M, Solomon C, Burke A. Comparison of necropsy findings in patients with sarcoidosis dying suddenly from cardiac sarcoidosis versus dying suddenly from other causes. Am J Cardiol. 2009;104(4):571-577.

24. Papageorgiou N, Providencia R, Bronis K, et al. Catheter ablation for ventricular tachycardia in patients with cardiac sarcoidosis: a systematic review. Europace. 2018;20(4):682-691. 
25. Koplan BA, Soejima K, Baughman K, Epstein LM, Stevenson WG. Refractory ventricular tachycardia secondary to cardiac sarcoid: electrophysiologic characteristics, mapping, and ablation. Heart Rhythm. 2006;3(8):924-929.

26. Dukkipati SR, d'Avila A, Soejima K, et al. Long-term outcomes of combined epicardial and endocardial ablation of monomorphic ventricular tachycardia related to hypertrophic cardiomyopathy. Circ Arrhythm Electrophysiol. 2011;4(2):185-194.

27. Ueda A, Fukamizu S, Soejima K, et al. Clinical and electrophysiological characteristics in patients with sustained monomorphic reentrant ventricular tachycardia associated with dilated-phase hypertrophic cardiomyopathy. Europace. 2012;14(5):734-740.

28. Santangeli P, Di Biase L, Lakkireddy D, et al. Radiofrequency catheter ablation of ventricular arrhythmias in patients with hypertrophic cardiomyopathy: safety and feasibility. Heart Rhythm. 2010;7(8):1036v1042.

29. Nademanee K, Veerakul G, Chandanamattha $P$, et al. Prevention of ventricular fibrillation episodes in Brugada syndrome by catheter ablation over the anterior right ventricular outflow tract epicardium. Circulation. 2011;123(12):1270-1279.

30. Fernandes GC, Fernandes A, Cardoso R, et al. Ablation strategies for the management of symptomatic Brugada syndrome: a systematic review. Heart Rhythm. 2018;15(8):1140-1147.

31. Yokokawa M, Desjardins B, Crawford T, Good E, Morady F, Bogun F. Reasons for recurrent ventricular tachycardia after catheter ablation of post-infarction ventricular tachycardia. J Am Coll Cardiol. 2013;61(1):66-73.

32. Kaltenbrunner W, Cardinal R, Dubuc M, et al. Epicardial and endocardial mapping of ventricular tachycardia in patients with myocardial infarction. Is the origin of the tachycardia always subendocardially localized? Circulation. 1991;84(3):1058-1071.

33. Hayashi T, Liang JJ. Epicardial ventricular tachycardia in ischemic cardiomyopathy: prevalence, electrophysiological characteristics, and long-term ablation outcomes. J Cardiovasc Electrophysiol. 2018;29(11):1530-1539.

34. Sarkozy A, Tokuda M, Tedrow UB, et al. Epicardial ablation of ventricular tachycardia in ischemic heart disease. Circ Arrhythm Electrophysiol. 2013;6(6):1115-1122.

35. Bogun FM, Desjardins B, Good E, et al. Delayed-enhanced magnetic resonance imaging in nonischemic cardiomyopathy: utility for identifying the ventricular arrhythmia substrate. J Am Coll Cardiol. 2009;53(13):1138-1145.

36. Piers SR, Zeppenfeld K. Imaging-guided ventricular tachycardia ablation. Arrhythm Electrophysiol Rev. 2013;2(2):128-134.

37. Russo RJ, Costa HS, Silva PD, et al. Assessing the risks associated with MRI in patients with a pacemaker or defibrillator. N Engl J Med. 2017;376(8):755-764.

38. Nazarian S, Hansford R, Rahsepar AA, et al. Safety of magnetic resonance imaging in patients with cardiac devices. $N$ Engl J Med. 2017;377:2555-2564.

39. Dickfeld T, Tian J, Ahmad G, et al. MRI-guided ventricular tachycardia ablation: integration of late gadolinium-enhanced 3D scar in patients with implantable cardioverter-defibrillators. Circ Arrhythm Electrophysiol. 2011;4(2):172-184.

40. Lamberti F, Calo L, Pandozi C, et al. Radiofrequency catheter ablation of idiopathic left ventricular outflow tract tachycardia: utility of intracardiac echocardiography. J Cardiovasc Electrophysiol. 2001;12(5):529-535.

41. BerruezoA, Mont L,NavaS, Chueca E, Bartholomay E, Brugada J. Electrocardiographic recognition of the epicardial origin of ventricular tachycardias. Circulation. 2004;109(15):1842-1847.
42. Bazan V, Gerstenfeld EP, Garcia FC, et al. Site-specific twelve-lead ECG features to identify an epicardial origin for left ventricular tachycardia in the absence of myocardial infarction. Heart Rhythm. 2007;4(11):1403-1410.

43. Valles E, Bazan V, Marchlinski FE. ECG criteria to identify epicardial ventricular tachycardia in nonischemic cardiomyopathy. Circ Arrhythm Electrophysiol. 2010;3(1):63-71.

44. Tzou WS, Nguyen DT, Aleong RG, et al. Endocardial electrogram characteristics of epicardial ventricular arrhythmias. J Cardiovasc Electrophysiol. 2013;24(6):649-654.

45. Hutchinson MD, Gerstenfeld EP, Desjardins B, et al. Endocardial unipolar voltage mapping to detect epicardial ventricular tachycardia substrate in patients with nonischemic left ventricular cardiomyopathy. Circ Arrhythm Electrophysiol. 2011;4(1):49-55.

46. Soto-Becerra R, Bazan V, Bautista W, et al. Ventricular tachycardia in the setting of chagasic cardiomyopathy: use of voltage mapping to characterize endoepicardial nonischemic scar distribution. Circ Arrhythm Electrophysiol. 2017;10(11).

47. Polin GM, Haqqani H, Tzou W, et al. Endocardial unipolar voltage mapping to identify epicardial substrate in arrhythmogenic right ventricular cardiomyopathy/dysplasia. Heart Rhythm. 2011;8(1):76-83.

48. Morady F, Strickberger A, Man KC, et al. Reasons for prolonged or failed attempts at radiofrequency catheter ablation of accessory pathways. J Am Coll Cardiol. 996;27(3):683-689.

49. Schweikert RA, Saliba WI, Tomassoni G, et al. Percutaneous pericardial instrumentation for endo-epicardial mapping of previously failed ablations. Circulation. 2003;108(11):1329-1335.

50. Valderrabano M, Cesario DA, Ji S, et al. Percutaneous epicardial mapping during ablation of difficult accessory pathways as an alternative to cardiac surgery. Heart Rhythm. 2004;1(3):311-316.

51. Sacher F, Wright M, Tedrow UB, et al. Wolff-ParkinsonWhite ablation after a prior failure: a 7-year multicentre experience. Europace. 2010;12(6):835-841.

52. Scanavacca MI, Sternick EB, Pisani C, et al. Accessory atrioventricular pathways refractory to catheter ablation: role of percutaneous epicardial approach. Circ Arrhythm Electrophysiol. 2015;8(1):128-136.

53. Sternick EB, Faustino M, Correa FS, Pisani C, Scanavacca MI. Percutaneous Catheter Ablation of Epicardial Accessory Pathways. Arrhythm Electrophysiol Rev. 2017;6(2):80-84.

54. Cox JL, Ad N, Palazzo T, et al. Current status of the Maze procedure for the treatment of atrial fibrillation. Semin Thorac Cardiovasc Surg. 2000;12(1):15-19. https:/ / www.ncbi. nlm.nih.gov/pubmed/10746917

55. Wolf RK, Schneeberger EW, Osterday R, et al. Video-assisted bilateral pulmonary vein isolation and left atrial appendage exclusion for atrial fibrillation. J Thorac Cardiovasc Surg. 2005;130(3):797-802.

56. Damiano RJ, Jr., Schwartz FH, Bailey MS, et al. The Cox maze IV procedure: predictors of late recurrence. J Thorac Cardiovasc Surg. 2011;141(1):113-121.

57. Henn MC, Lancaster TS, Miller JR, et al. Late outcomes after the Cox maze IV procedure for atrial fibrillation. J Thorac Cardiovasc Surg. 2015;150(5):1168-1176, 1178.e1161-1162.

58. Musharbash FN, Schill MR, Sinn LA, et al. Performance of the Cox-maze IV procedure is associated with improved long-term survival in patients with atrial fibrillation undergoing cardiac surgery. J Thorac Cardiovasc Surg. 2018;155(1):159-170.

59. de Asmundis C, Chierchia GB, Mugnai G, et al. Midterm clinical outcomes of concomitant thoracoscopic epicardial 
and transcatheter endocardial ablation for persistent and long-standing persistent atrial fibrillation: a single-centre experience. Europace. 2017;19(1):58-65.

60. McKinnie J. The convergent procedure-a standardised and anatomic approach addresses the clinical and economic unmet needs of the persistent atrial fibrillation population. Arrhythmia Electrophysiol Rev. 2013;2(2):145-148.

61. Civello KC SC, Boedefeld W. Combined endocardial and epicardial ablation for symptomatic atrial fibrillation: single center experience in $100+$ consecutive patients. J Innov Card Rhythm Manage. 20131-20137.

62. Gilligan DM JC, Bundy GM. Multidisciplinary collaboration for the treatment of atrial fibrillation: convergent procedure outcomes from a single center. J Innov Card Rhythm Manage. 2013;4:1396-1403.

63. Gersak B, Jan M. Long-term success for the convergent atrial fibrillation procedure: 4-year outcomes. Ann Thorac Surg. 2016;102(5):1550-1557.

64. Zembala M, Filipiak K, Kowalski O, et al. Staged hybrid ablation for persistent and longstanding persistent atrial fibrillation effectively restores sinus rhythm in long-term observation. Arch Med Sci. 2017;13(1):109-117.

65. Luo X, Li B, Zhang D, Zhu J, Qi L, Tang Y. Efficacy and safety of the convergent atrial fibrillation procedure: a meta-analysis of observational studies. Interact Cardiovasc Thorac Surg. 2019;28(2):169-176.

66. La Meir M, Gelsomino S, Luca F, et al. Minimally invasive surgical treatment of lone atrial fibrillation: early results of hybrid versus standard minimally invasive approach employing radiofrequency sources. Int J Cardiol. 2013;167(4):1469-1475.

67. Schuessler RB, Lee AM, Melby SJ, et al. Animal studies of epicardial atrial ablation. Heart Rhythm. 2009;6(12 Suppl):S41-S45.
68. Xu J, Luc JG, Phan K. Atrial fibrillation: review of current treatment strategies. J Thorac Dis. 2016;8(9):E886-E900.

69. Piorkowski C, Kronborg M, Hourdain J, et al. Endo-/epicardial catheter ablation of atrial fibrillation: feasibility, outcome, and insights into arrhythmia mechanisms. Circ Arrhythm Electrophysiol. 2018;11(2):e005748.

70. Di Biase L, Burkhardt JD, Pelargonio G, et al. Prevention of phrenic nerve injury during epicardial ablation: comparison of methods for separating the phrenic nerve from the epicardial surface. Heart Rhythm. 2009;6(7):957-961.

71. Lee RJ, Kalman JM, Fitzpatrick AP, et al. Radiofrequency catheter modification of the sinus node for "inappropriate" sinus tachycardia. Circulation. 1995;92(10):2919-2928.

72. Jacobson JT, Kraus A, Lee R, Goldberger JJ. Epicardial/ endocardial sinus node ablation after failed endocardial ablation for the treatment of inappropriate sinus tachycardia. J Cardiovasc Electrophysiol. 2014/03/01 2014;25(3): 236-241.

73. Koplan BA, Parkash R, Couper G, Stevenson WG. Combined epicardial-endocardial approach to ablation of inappropriate sinus tachycardia. J Cardiovasc Electrophysiol. 2004;15(2):237-240.

74. Killu AM, Syed FF, Wu P, Asirvatham SJ. Refractory inappropriate sinus tachycardia successfully treated with radiofrequency ablation at the arcuate ridge. Heart Rhythm. 2012;9(8):1324-1327.

75. Gianni C, Di Biase L, Mohanty S, et al. Catheter ablation of inappropriate sinus tachycardia. J Interv Card Electrophysiol. 2016;46(1):63-69.

76. Rodriguez-Manero M, Kreidieh B, Al Rifai M, et al. Ablation of inappropriate sinus tachycardia: a systematic review of the literature. JACC Clin Electrophysiol. 2017;3(3):253-265.

77. Olshansky B, Sullivan RM. Inappropriate sinus tachycardia. Europace. 2019;21(2):194-207. 